\title{
The Construction of Minimum Variables Set for Energy Prediction Models of Office Buildings
}

\author{
Mingya Zhu ${ }^{1}$, Yiqun Pan ${ }^{1}$, Zhizhong Huang ${ }^{2}$ \\ ${ }^{1}$ School of Mechanical Engineering, Tongji University, Shanghai, China \\ ${ }^{2}$ Sino-German College of Applied Sciences, Tongji University, Shanghai, \\ China
}

\begin{abstract}
A variety of building energy prediction models have been widely used in building performance optimization. While the high data requirements of most models present a barrier to effective building performance management. At the same time, the shortage of reasonable variable selection has resulted in reduced credibility and generalization of most model results. This study aims to deeply explore the indispensable variables of building energy prediction and put forward the concept of minimum variables sets for reasonably accurate building energy prediction in the condition of limited data information. With application of feature dimension reduction methods to a building performance database, the hierarchical minimum variables sets are constructed for multiple building energy prediction objects. By means of two kinds of energy models, the accuracy of the minimum variables sets is analysed and evaluated to provide theoretical basis and data support for practically appropriate trade-off of limited data and acceptable requisition of building energy prediction.
\end{abstract}

\section{Introduction}

There currently existed two main kinds of building energy predictive models: forward and data-driven methods (Foucquier et al., 2013). The forward approach is to predict the building energy use of a specified model with known physical description of energy system (e.g. thermal performance and system parameters) and known building design parameters (e.g. internal gains, thermostat settings), as well as climate conditions. The forward modelling procedure and its application is quite unified in the related researches, and has developed into several detailed building energy simulation tools, such as eQUEST and EnergyPlus. Unlike the forward simulation, the data-driven model applies statistical methods to determine a mathematical description of building energy system when above building factors and energy use are known and measured. According to different used statistic method, common data-driven models include regression model, time series model, artificial neural network, support vector machine, and calibrated simulation.

A variety of building energy prediction models have been widely used in the optimized building design and efficient building operation. However, both forward and data-driven energy prediction models have high requirements of model data base. The conflicts between high requirements and limited availability of actual data present a barrier to effective building performance management.

Previous researches on building energy use analysis have mostly used the sensitivity analysis routine to explore the influence of some building parameters. And relative studies have focused on either partial building parameters (e.g. constant weather parameter or HVAC system type) or sub-metering building energy consumption (e.g. heating or cooling consumption), even a specific actual building case study (Neto et al. 2008, Santamouris et al. 1996, Yang et al. 2015), with limitation of variable definition or database shortage for full-scale variable coverage. However, accurate model variables and data information are crucial to fully achieve potential performance of building prediction models. The shortage of reasonable variable selection has resulted in reduced credibility and generalization of most researches. In this case, most of current modellers just choose building parameters available or selected by others as the model variables. Current variable selection process makes the model data basis of related researches different from each other. The corresponding conclusions make little sense and reference for others with different goals of building energy prediction.

In the few present researches on variable selection for building energy prediction models, most are developed by data mining researchers for the application of several statistical methods, especially the data-driven models such as ANN and SVM, in the building energy prediction field (Olofsson et al. 2001, Lv et al. 2010, Zhao et al. 2010).

After a deep understanding of the variable selection for building energy models, we found it similar to the feature selection problem in the statistical analysis. The nature of feature dimension reduction is to find suitable lower dimensional mapping to represent the initial high dimensional space.

Based on this, the study considered to explore the fundamental question: which building parameters are the indispensable variables of building energy prediction models? Besides, commonly used building energy models were used to explore another fundamental question, how to accurately predict building energy consumption with the limited building variables? On the 
basis of a building performance database (BPD) including about 10,000 building models, this study regarded the high dimensional data space built by the building variables and their corresponding energy consumption in the BPD as a reflection of the complicated mapping relationship between building parameters and energy consumption. In the core part, we applied the feature dimension reduction methods to the primary high dimensional space, then proposed and built the minimum variables set for building energy prediction. The minimum variables set includes the most essential variables for building energy consumption prediction. The model using minimum variables set as its model variables could achieve accurate building energy consumption with less training or modelling data.

\section{Methods}

In this study, there obviously have two key parts. First is the sufficient and full-scale BPD as the data basis, the second is reasonable feature dimension reduction process. Accordingly, in the first part, the case design and construction process are important to guarantee the coverage and comprehensiveness of the BPD to ensure the generalization of the following feature selection research. To keep this paper reasonably concise, the first part had been published in the Proceeding of ASim 2018 (Zhu M. et al. 2018).

This paper will focus on the feature dimension reduction application in the prepared BPD. Similarly, the suitable feature dimension reduction methods and selection criteria are essential to obtain reasonable and acceptable variables sets for building energy prediction.

Feature dimension reduction is a most important preprocessing method for high-dimensional data mining models. A basic hypothesis of feature dimension reduction problem is that high-dimensional data samples are actually, at least approximatively located on a lowerdimensional manifold due to the common redundancy of data information. The feature reduction problem has different terms in several areas, e.g. named feature extraction in pattern recognition researches, and data compression in information theory. While in essence, it is to find the lower-dimensional projection mapping of the primary high dimension space and simultaneously keep the high-dimensional spatial characteristics as far as possible by means of removing data redundancies and enhancing useful features. Given the mixed data feature of the prepared BPD, we considered the following two methods: 1) Principle component analysis based on data transformation, and 2) Max Relevance Min Redundancy filtration (MRMR) to obtain different minimum variables sets for multi-scale building energy prediction.

The application of common feature selection methods in data mining researches often has uniform criteria out of mathematical rationality. While the selected variables under a uniform rule are always difficult to meet the demands of input requirement, data availability and time-effectiveness of practical models development. Meanwhile, there may be discrepancy between the statistical rationality of mathematical methodology and accuracy requirement of practical application. Given this, we considered to propose the more universal and flexible hierarchical variables sets with different dimensions under quantitative gradient criterion, which will be the powerful data support for reasonable variable and model selection in the building energy prediction field.

\section{The prepared BPD and its mixed data feature}

Based on the proposed case design method and parallel modeling, the simulated BPD can basically reflect the complex mapping relation among building variables and office building energy consumption from the statistical perspective.

The prepared BPD comprised of 9,750 building cases having high-dimensional mixed 17 building variables and the corresponding multiple time-scale building energy consumption. Its building variables include weather parameters, building shape, envelope, internal load, HVAC system and operation schedule, see in Table 1. The range of each variable is determined according to ASHRAE or national codes and actual office building situation in the cities located in the north hemisphere. The results include whole building energy consumption, sub-metering consumption (e.g. cooling, heating and lighting etc.) and daily consumption in the form of energy utility index (EUI). The BPD construction process has been detailed in Zhu M. et al. 2018.

Building variables of the BPD include two types: numeric and non-numeric variables. The numeric variable analysis has been mature in statistics. While for non-numeric variables, it's difficult to directly obtain their distance or similarity metrics due to the noncontinuity. Current metrics for categorical variables have often applied the distribution of variables, e.g. value difference metric (VDM) and simple matching distance (SMD) (Zhao L. et al. 2016). In this case, one route for mixed data feature reduction is to do the quantized transformation of the qualitative variables before the conventional feature selection, e.g. the PRINQUAL process before PCA (Zhang X. 2016). The other route is to define new similarity metrics for mixed data and use the conventional feature selection rules to obtain a feature subset, e.g. the combined polychoric and polyserial correlation (Kolenikov S. et al. 2004).

Here we've applied the first technical route to the BPD for feature dimension reduction and construction of minimum variables sets for building energy prediction. In statistics, feature reduction methods are generally divided into two types: 1) feature extraction and 2) feature selection. According to the mentioned research route, both of them are introduced in the following section.

\section{Principle component analysis for mixed data based on data transformation}

Principle component analysis (PCA) is the most commonly used feature dimension reduction method, which is belong to the feature extraction type. The key of feature extraction is to achieve the projection mapping of primary high-dimension space to a lower-dimension space. PCA explores to explain the variance-covariance 
structure of multiple variables by means of several principle components based on the greatest variability rule. The motivation behind this problem is that the directions of greatest variability give "most information" about the configuration of the data in the primary space (Kolenikov S. et al. 2004). This rule means that the projections of primary samples to the lower-dimensional hyperplane are separate as far as possible to obtain the maximum sample variance. (Zhou Z. 2016)

PRINQUAL procedure (SAS, 2003) performs principal component analysis of qualitative, quantitative or mixed data, combining the data transformation procedure with traditional PCA. It uses alternating least square methods to keep the covariance or correlation matrix of transformed variables at an optimal level, including both linear and non-linear transformation. It has three optional rules for transformation methods, maximum total variance (MTV), minimum generalized variance (MGV) and maximum average correlation (MAC). The PRINQUAL iterations produce a set of transformed quantitative variables for the following data mining work.

For the BPD, the mixed PCA has been achieved by calling PRINQUAL and PRINCOMP procedures (SAS, 2003) to separately complete the data transformation and conventional PCA analysis. The above feature extraction process will obtain the minimum variables set for office building energy prediction.

Max Relevance Min Redundancy (MRMR) filtration based on feature relativity

Different from the feature extraction, the other type of feature dimension reduction is feature selection, the key of which is to directly select variable subsets to rebuild a lower-dimension space. The earliest feature selection has been applied in the supervised learning researches and focused on the feature relativity. The key of feature filtering is to find the feature subset having the maximum relevance to the decision variable, and at the same time the redundancy among them is as small as possible. The classic Max-Relevance Min-Redundancy (MRMR) method uses this theory to select features (Peng H.C. et al. 2005). In MRMR method, there have some criterion to decide the relevant and redundant features. The relevance between features and the dependent variable reflects the contribution of each feature to decision variable and can be used to find the relevant features by t-test or Fisher rule. While, the correlation among features reflects the similarity among features and can be used to exclude the redundant features by mutual information or Pearson correlation coefficient (PCC).

For the BPD, we have used the PCC to be the feature relativity metric and applied the MRMR method to construct the minimum variables sets for office building energy consumption prediction. As shown in equation (1), the correlation coefficient, $\rho_{(\xi, \eta)}$ is the correlation of two variables, $\operatorname{cov}(\xi, \eta)$, divided by the product of their standard deviation, $\sqrt{\operatorname{var}(\xi)}$ and $\sqrt{\operatorname{var}(\eta)}$. Within the range of $-1 \sim 1$, the absolute $\rho_{(\xi, \eta)}$ is closer to 1 , the two variables are more relevant. In the feature selection process, we'll keep the variables having the most relevance to office building energy consumption and the least correlation among themselves.

$$
\rho_{(\xi, \eta)}=\frac{\operatorname{cov}(\xi, \eta)}{\sqrt{\operatorname{var}(\xi)} * \sqrt{\operatorname{var}(\eta)}}
$$

\section{Proposed minimum variables set construction method}

With the above two method options, the supervised feature dimension reduction process is separately conducted to the high-dimensional building variables and the corresponding building energy consumption in the BPD. In this way, the representativeness of each building variable on the complicated mapping relationship between high-dimensional building variables and office building energy consumption could be analyzed and ranked.

For the sake of the uncertain gap between the theoretically statistical rationality and practically applicant accuracy, we considered not to directly use traditional feature reduction process and simply apply specific statistical index, e.g. variance resolution to judge the reliability of results. Strictly speaking, the practical accuracy of the resulted lower-dimensional feature space needs to be validated by its following application of building energy prediction through the way of being input variables for common building energy models.

Under the above consideration, we analyzed the similarities and differences of the two groups of feature reduction results separately through feature extraction and feature selection method. The comparison indicates a great degree of similarity between two resulted variables sets. Most of them are inter-inclusive, only a very few variables are exclusive by the other group or the prioritization ranking is slightly different. The detailed comparison process is not presented in this paper for the sake of brevity. After this key step, the union of two groups are reserved to keep both feature reduction rules before the accuracy validation researches as the following exploration work in section Results.

To construct more feasible and universal variable sets for practical office building energy prediction application, we proposed different criterions for the application process of the two feature reduction methods with the conventional statistical requirement for feature selection as a baseline. As shown in Figure 1, each feature reduction method commonly has their own feature selection basis in the application process, which is determined by their own methodological rules. With the same selection basis of a feature reduction method, we can obtain different scales of lower-dimension variable sets under different selection criterions. For example, the selection basis of the PCA method are the ranking and the variance resolution of each eigenvalues. Traditional PCA application often keeps the variables who represent about $80 \%$ of variance resolution for primary high-dimensional space as the principle variables. If we change the proportion of the selection criterion from $80 \%$ to $85 \%$ or $75 \%$, the achieved variable sets will separately have more or less variables 


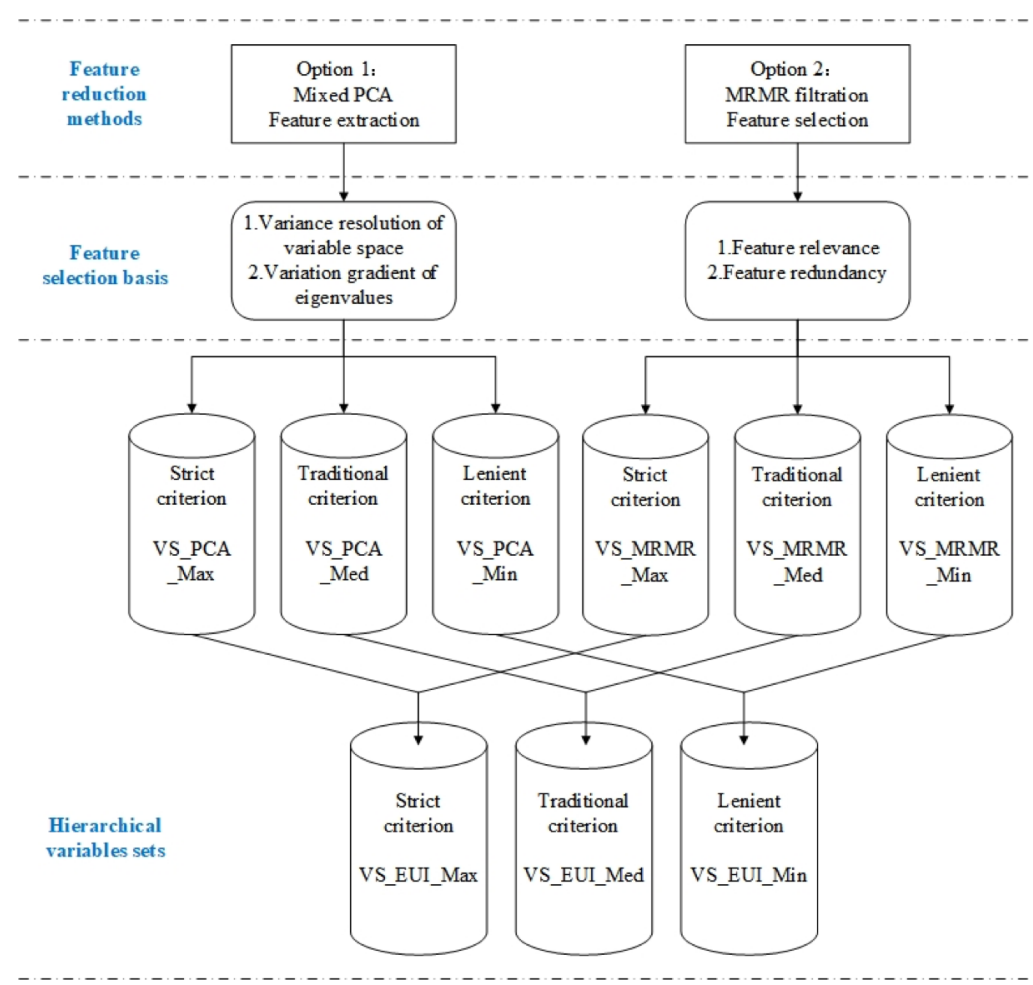

Figure 1: The proposed minimum variable sets construction method

than the traditional PCA application results. As to the MRMR filtration method, the different criterions for its selection basis can be reflected by different limiting values of the acceptable correlation coefficients. In this case, under three levels of criterions, we can separately obtain Variable Sets of Maximum, Medium, and Minimum scales, called VS_MethodName_Max, VS_MethodName_Med, and VS_MethodName_Min in Figure 1. According to the resulted prioritization ranking of building variables in both methods, this study combined the two groups of feature reduction results and merged them to construct the dimensionally hierarchical minimum variables sets for office building energy prediction with the quantitative criteria, called VS_EUI_Max, VS_EUI_Med, and VS_EUI_Min in Figure 1.

\section{Results}

The annual energy consumption is one of the most important temporal scale in majority of the building energy prediction researches for both existed building efficient operation and new building optimized design. The whole building energy consumption and the submetering heating/cooling energy consumption are the two main predictive energy objects for building energy management on the yearly scale. Given that, this study applied the proposed minimum variables set construction method to the mentioned BPD including 17 primary variables and the corresponding multi-dimensional energy consumption results. In this way, we mainly analyzed and selected the indispensable building variables from the 17 primary variables (see in Table 1) for annual and sub-metering heating/cooling energy consumption prediction to separately construct the minimum variables sets for each predictive energy object.
This section will firstly introduce the feature reduction method application process using the whole building annual energy consumption object as an example. Then the application of achieved variables sets for common building energy prediction model is explored and evaluated to explain that how to achieve reasonable building energy prediction with limited model variables.

Table 1: Primary variables design for BPD

\begin{tabular}{|c|c|c|}
\hline $\begin{array}{c}\text { Numerical } \\
\text { variable }\end{array}$ & Description & Range \\
\hline v1_sat & $\begin{array}{c}\text { Summer average } \\
\left.\text { temperature [ }{ }^{\circ} \mathrm{C}\right]\end{array}$ & $16.0 \sim 31.0$ \\
\hline v2_wat & $\begin{array}{c}\text { Winter average } \\
\left.\text { temperature [ }{ }^{\circ} \mathrm{C}\right]\end{array}$ & $-11.0 \sim 23.2$ \\
\hline v3_tat & $\begin{array}{c}\text { Transition average } \\
\left.\text { temperature [ }{ }^{\circ} \mathrm{C}\right]\end{array}$ & $4.5 \sim 24.9$ \\
\hline v4_sarh & $\begin{array}{c}\text { Summer average } \\
\text { relative humidity }\end{array}$ & $0.28 \sim 0.88$ \\
\hline v5_bsc & $\begin{array}{c}\text { Building Shape } \\
\text { Coefficient }\end{array}$ & $0.10 \sim 0.50$ \\
\hline v6_wwr & Window wall ratio & $0.10 \sim 1.00$ \\
\hline v7_ohtc & $\begin{array}{c}\text { Overall Heat Transfer } \\
\text { Coefficient, OHTC } \\
\text { [W/m }{ }^{2} \text { ] }\end{array}$ & $5.0 \sim 35.0$ \\
\hline v8_lpd & $\begin{array}{c}\text { Lighting power } \\
\text { density [W/m }{ }^{2} \text { ] }\end{array}$ & $10.0 \sim 20.0$ \\
\hline v9_ppd & $\begin{array}{c}\text { People power density } \\
{\left[\mathrm{m}^{2} / \mathrm{p}\right]}\end{array}$ & $2.0 \sim 10.0$ \\
\hline v10_epd & $\begin{array}{c}\text { Equipment power } \\
\text { density [W/m }{ }^{2} \text { ] }\end{array}$ & $10.0 \sim 20.0$ \\
\hline
\end{tabular}




\begin{tabular}{|c|c|c|}
\hline v11_sidt & $\begin{array}{c}\text { Summer Indoor design } \\
\text { temperature }\left[{ }^{\circ} \mathrm{C}\right]\end{array}$ & $22.0 \sim 28.0$ \\
\hline v12_widt & $\begin{array}{l}\text { Winter Indoor design } \\
\text { temperature }\left[{ }^{\circ} \mathrm{C}\right]\end{array}$ & $15.0 \sim 22.0$ \\
\hline v13_bldgA & $\begin{array}{l}\text { Building area } \\
{\left[\times 10^{3} \mathrm{~m}^{2}\right]}\end{array}$ & $2 \sim 10$ \\
\hline $\begin{array}{c}\text { Non- } \\
\text { numerical } \\
\text { variable }\end{array}$ & Description & Range \\
\hline \multirow{5}{*}{ v14_HVAC } & All zones:CAV & A0 \\
\hline & All zones:VAV & A1 \\
\hline & All zones:FCU+OA & A2 \\
\hline & $\begin{array}{c}\text { Inner :VAV } \\
\text { Perimeter:FCU+OA }\end{array}$ & A3 \\
\hline & All zones:VRV & A4 \\
\hline \multirow{6}{*}{ v15_plant } & CentiChiller \& Boiler & P0 \\
\hline & ScrewChiller \& Boiler & P1 \\
\hline & $\begin{array}{l}\text { Absorption chiller\& } \\
\text { Boiler }\end{array}$ & P2 \\
\hline & $\begin{array}{l}\text { Ground source heat } \\
\text { pump }\end{array}$ & $\mathrm{P} 3$ \\
\hline & Air source heat pump & P4 \\
\hline & $\begin{array}{c}\text { CentiChiller \& Heat } \\
\text { pump }\end{array}$ & P5 \\
\hline v16_tspt & Variable speed pumps & $\mathrm{Y} / \mathrm{N}$ \\
\hline v17_schd & Operation schedules & $\begin{array}{l}\mathrm{High} / \mathrm{Std} / \text { Low } \\
\text { usage }\end{array}$ \\
\hline
\end{tabular}

and obtained different minimum variables set with hierarchical dimensions for annual EUI prediction, as shown in Table 2.

As for the annual energy consumption prediction, the most significant variables are the HVAC system type, building operation schedule, weather files, building shape and occupants density, in turn. When applying the same research route to cooling/heating energy consumption prediction object, the most important variables are HVAC/energy source system type, floor area, weather files, internal load density, building operation schedule and envelope properties, in turn. And the application process will not be presented in details. To sum up, the result indicates that the most stringent feature selection criteria select the most dimensions of variables (12/13), that those selected by the conventional criteria are medium (8/9), and that those selected by the loosest selection criterion have the least dimensions (6/7), where the number of variables from large to small means that fewer variables can be utilized to predict office building energy consumption when the selection criteria are looser.

Table 2: Minimum variables sets for annual EUI prediction.

\begin{tabular}{|c|c|c|c|}
\hline Sets & VS_EUI_Max & VS_EUI_Med & VS_EUI_Min \\
\hline $\begin{array}{l}\text { Selected } \\
\text { variables }\end{array}$ & $\begin{array}{c}\text { v14_HVAC } \\
\text { v15_plant } \\
\text { v16_trsp } \\
\text { v1_sat } \\
\text { v2_wat } \\
\text { v3_tat } \\
\text { v5_bsc } \\
\text { v13_bldgA } \\
\text { v17_schd } \\
\text { v9_ppd } \\
\text { v6_wwr } \\
\text { v11_sidt } \\
\text { v8_lpd }\end{array}$ & $\begin{array}{c}\text { v14_HVAC } \\
\text { v1_sat } \\
\text { v2_wat } \\
\text { v5_bsc } \\
\text { v17_schd } \\
\text { v9_ppd } \\
\text { v6_wwr } \\
\text { v11_sidt } \\
\text { v8_lpd }\end{array}$ & $\begin{array}{c}\text { v14_HVA } \\
\text { C } \\
\text { v17_schd } \\
\text { v1_sat } \\
\text { v2_wat } \\
\text { v5_bsc } \\
\text { v6_wwr } \\
\text { v9_ppd }\end{array}$ \\
\hline $\begin{array}{l}\text { Variable } \\
\text { number }\end{array}$ & 13 & 9 & 7 \\
\hline $\begin{array}{l}\text { Selection } \\
\text { criteria for } \\
\text { PCA } \\
\text { method }\end{array}$ & $\begin{array}{c}\text { Whole } \\
\text { variables for } \\
80 \% \\
\text { variance } \\
\text { explained }\end{array}$ & $\begin{array}{c}\text { Most loaded } \\
\text { variables for } \\
80 \% \\
\text { variance } \\
\text { explained }\end{array}$ & $\begin{array}{c}\text { Most } \\
\text { loaded } \\
\text { variables } \\
\text { for } 70 \% \\
\text { variance } \\
\text { explained }\end{array}$ \\
\hline $\begin{array}{l}\text { Selection } \\
\text { criteria for } \\
\text { MRMR } \\
\text { method }\end{array}$ & $\begin{array}{c}\text { Relevance }> \\
0.05 \text { and } \\
\text { have } \\
\text { redundancy }\end{array}$ & $\begin{array}{l}\text { Relevance }> \\
0.05 \text { and no } \\
\text { redundancy }\end{array}$ & $\begin{array}{l}\text { Relevance> } \\
0.1 \text { and no } \\
\text { redundancy }\end{array}$ \\
\hline
\end{tabular}

Minimum variables sets construction for annual building energy consumption

With the BPD as the data basis, we use the 17 primary variables as the feature select range and the corresponding building energy consumption EUI results as the dependent variable to conduct a supervised feature reduction. Through the data transformation and PCA process, we can get the eigenvectors of principal components and the corresponding eigenvalues of the mapped low-dimensional space. By using hierarchical extraction criterion in varying proportions of explained variance for PCA method, different scales of variables are separately selected for minimum variables sets of annual EUI prediction of office buildings. At the same time, based on the correlation analysis between building variables and annual EUI and among themselves, the MRMR method also can set different threshold relevance and redundancy coefficients to select building variables with different scales.

From the feature reduction results of the two mentioned methods, it's obvious that, at each criterion level, the selected variables by different methods are mostly the same and also have few different variables. After comparing the representative degree ranks of building variables for annual EUI under different feather selection criteria, we merged the two stratified selection results

Minimum variables sets application for building energy consumption prediction

So far, this study has applied suitable feature reduction methods and constructed dimensionally hierarchical minimum variables sets for the annual energy prediction of office buildings. With the statistical feature reduction criterion in the mentioned methods, the variable selection results obviously have sufficient mathematical 
rationality. So, the next important question is, how to reasonably use the minimum variables sets to accurately predict office building energy consumption? This section will try to analyze the application feasibility and accuracy of the achieved variables sets for building energy prediction from the perspective of two types of commonly used building energy models, forward and data-driven model.

For data-driven models, the model structure is flexible according to their model variables. So, we can directly apply the minimum variables sets to data-driven models by using the selected variables as the model independent variables. When use the hierarchical variables, the less model variables mean the decreased training data for data-driven model development. For forward models, the situation is relatively complex. Their abundant outputs benefit from detailed and full-scale input parameters and modular calculation procedure. This means the relatively fixed model structure and model variables. If apply to forward models, we can try an indirect way by just referring to the representative degree ranks of building variables and default the other variables excluded from the minimum variables sets according to common building design standards to ensure the model feasibility.

Based on feasibility analysis, this study has further explored the accuracy when the achieved minimum variables sets are used as model variables to predict building energy consumption of office building. Without plenty of actual building information or energy use data to directly develop data-driven models and evaluate the prediction accuracy, we considered to use part of the BPD to do this work.

With the high-dimensional space theory, the whole designed BPD gives a statistical perspective to describe the complex relationship between building variables and energy consumption, being the basis of the minimum variables sets exploration for building energy prediction. Without considering the inherent error caused by the used software itself, the BPD can cover enough variety of building factors. Based on this understanding, we can use part of the BPD to validate the accuracy of the minimum variables sets under different building situations from the sight of forward models. About $10 \%$ of the BPD (almost 1000 EnergyPlus models) are used as the base models with all 17 building variables accurate. Then we set the variables excluded from the minimum variables sets as default in the 1000 models and compared the building energy consumption EUI of the changed models with the base models to obtain the prediction error caused by limited building variables. The prediction error of the 1000 models under the application of VS_EUI_Max including 13 variables are distributed normally, see in Figure 2. The result indicates that, the prediction error of VS_EUI_Max for variant office buildings is mainly (95\%) mainly limited to $10 \sim 10 \%$, and the most error is $-12 \%$ and $15 \%$, which is acceptable for conventional annual building energy prediction.
After the validation research of the constructed minimum variables sets by EnergyPlus models, this study has additionally explored to build the hierarchical relationship among the minimum variable set, the feature selection criteria and the model prediction accuracy with both forward and data-driven models.

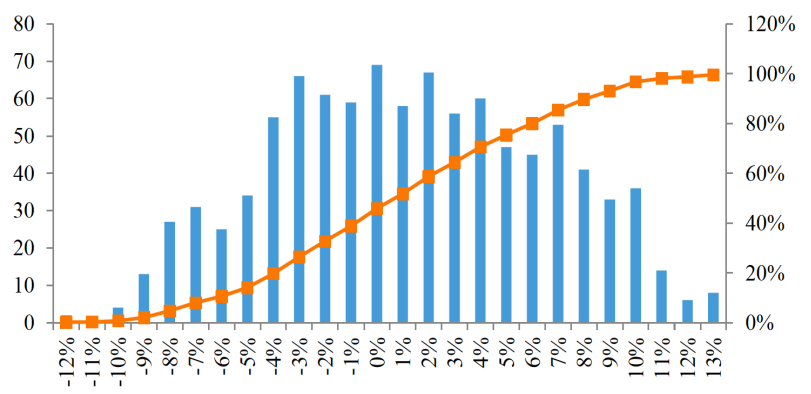

Figure 2: The prediction error distribution of VS_EUI_Max using EnergyPlus models.

Based on building information and the measured energy consumption of two actual office buildings separately located in Shanghai and California, within the designed BPD variable ranges, we developed the calibrated EnergyPlus models by actual building information as the baseline models and used the same way of the above validation research to gradually decrease the number of correct building variables according to the minimum variables sets constitution. At the same time, we established BP neural network models for annual EUI with hierarchical minimum variable set as model variables, trained by the BPD, and the developed ANN models are used predict the annual EUIs of the two actual buildings. With the error of the predicted EUI of two types of energy models, we obtained the graded impact of the minimum variable set with different selection criteria and dimensional scales on the accuracy of the energy consumption prediction model, see in Table 3.

Table 3: Hierarchical relationship among VS_EUI, selection criteria and prediction error of annual EUI prediction of actual office buildings.

\begin{tabular}{|c|c|c|c|}
\hline $\begin{array}{c}\text { Variable } \\
\text { number }\end{array}$ & VS_EUI_Max & VS_EUI_Med & VS_EUI_Min \\
\hline $\begin{array}{c}\text { Selection } \\
\text { criteria for } \\
\text { PCA } \\
\text { method }\end{array}$ & $\begin{array}{c}\text { Whole } \\
\text { variables for } \\
80 \% \text { variance } \\
\text { explained }\end{array}$ & $\begin{array}{c}\text { Most loaded } \\
\text { variables for } \\
80 \% \text { variance } \\
\text { explained }\end{array}$ & $\begin{array}{c}\text { Most loaded } \\
\text { variables for } \\
70 \% \text { variance } \\
\text { explained }\end{array}$ \\
\hline $\begin{array}{c}\text { Selection } \\
\text { criteria for } \\
\text { MRMR } \\
\text { method }\end{array}$ & $\begin{array}{c}\text { Relevance }>0.05 \\
\text { and have } \\
\text { redundancy }\end{array}$ & $\begin{array}{c}\text { Relevance }>0.05 \\
\text { and no } \\
\text { redundancy }\end{array}$ & $\begin{array}{c}\text { Relevance }>0.1 \\
\text { and no } \\
\text { redundancy }\end{array}$ \\
\hline $\begin{array}{c}\text { Prediction } \\
\text { error of } \\
\text { EnergyPlus }\end{array}$ & $-6 \% \sim 9 \%$ & $-8 \% \sim 11 \%$ & $-14 \% \sim 17 \%$ \\
\hline $\begin{array}{c}\text { Prediction } \\
\text { error of } \\
\text { ANN }\end{array}$ & $-11 \% \sim-1 \%$ & $-9 \% \sim 15 \%$ & $-13 \% \sim 24 \%$
\end{tabular}

The result indicates that, for the whole building annual EUI, the prediction error using minimum variables sets under the most stringent criteria as model variables is 
less than $10 \%$, and the prediction error of that under conventional criteria is less than $15 \%$, and when the loosest selection criterion is employed, the error increases to about less than $25 \%$. When it comes to the cooling sub-item, the corresponding prediction errors using the above three minimum variable sets are respectively within $5 \%$, around $5 \%$ and about $10 \%$, and the application process will not be presented in details.

\section{Discussion}

The built BPD is an important data basis of this study. Given the comprehensive consideration of cases number, computation efficiency and database complexity, we did some simplification for the primary building variable design, which may result in possible error of the achieved results in this study. For example, the building shape is limited to the regular square and rectangle, and the other shape, such as L shape may cause about $5 \%$ of prediction error through some case tests. And some variables are just on a relatively rough scale, like the system variables are on the HVAC or energy system type level, and the building envelope and operation schedule variables are on the scenario level according to common building design standards. The more specific parameters included in these variables are not studied. The error caused by above simplification process can also be analysed by some parametric uncertainty discussion in the future work, and the schedule variable may need to introduce related occupant behaviour to do more exploration. Also, the constructed BPD are comprised by plenty of calculated EnergyPlus cases and the software calculation error is not considered.

Owing to the limitation of forward model structure, the validation work of the constructed minimum variables sets is insufficient. In the predictive accuracy validation process, we can only check the predictive error distribution of VS_EUI_Max having the most selected variables. The two lower-dimensional minimum variable sets include some variables couldn't be defaulted, so they couldn't be directly used in the forward EnergyPlus models with fixed model input requirements. At the same time, without plenty of actual building data, the accuracy evaluation work of the minimum variables sets and the corresponding conclusion are also not enough to be directly useful support to other practical buildings. More actual buildings will be collected to analyze the applicability and uncertainty of the research results in the future.

\section{Conclusion}

Aim to solve the problem of using limited data to achieve reasonable and accurate building energy prediction, this study firstly put forward the concept of minimum variable set for building energy prediction models. Using a built full-sale BPD as a highdimensional space, we introduced feature dimension reduction method to solve the problem of variable selection in the building energy prediction and constructed the minimum variables sets for multiple building energy prediction objects, including annual whole building and heating/cooling energy consumption EUI. To get more feasible and universal variable selection for practical application, we constructed the hierarchical minimum variables sets, where the number of variables from large to small means that fewer variables can be utilized to predict building energy consumption when the selection criteria are looser.

With the two types of commonly used building energy models, this study has analyzed the application feasibility and further explored how to utilize above research results to implement reasonable and accurate building energy consumption with limited data. Based on the built BPD and actual building data, the prediction accuracy of the minimum variables sets is validated and evaluated by building the hierarchical relationship among the minimum variable set, the feature selection criteria and the model prediction accuracy, which can provide theoretical basis and data support for appropriate trade-off of limited basic data and higher requisition of office building energy prediction in practical applications.

\section{References}

Foucquier A, Robert S, Suard F, Stéphan L, Jay A(2013). State of the art in building modelling and energy performances prediction: A review. Renewable and Sustainable Energy Reviews 23, 272-288.

Kolenikov S., Angeles G. (2004), The Use of Discrete Data in PCA: Theory, Simulations, and Applications to Socioeconomic Indices:[Doctoral Thesis]. University of North Carolina

Lv J., Li X., Ding L., Jiang L.(2010), Applying principal component analysis and weighted support vector machine in building cooling load forecasting. 2010 International Conference on Computer and Communication Technologies in Agriculture Engineering, 434-437

Neto A.H., Fiorelli F.A.S. (2008), Comparison between detailed model simulation and artificial neural network for forecasting building energy consumption. Energy and Buildings 40 (12), 2169-2176

Olofsson T., Andersson S. (2001), Long-term energy demand predictions based on short-term measured data. Energy and Buildings 33 (2), 85-91

Peng H.C., Long F.H., Ding C. (2005), Feature selection based on mutual information: Criteria of maxdependency, max-relevance, and min-redundancy. IEEE Transactions on Pattern Analysis and Machine Intelligence 27(8):1226-1238

Santamouris M., Balaras C.A., Dascalaki E., Gaglia A.(1996), Energy Conservation and Retrofitting Potential in Hellentic Hotels. Energy and Buildings 24 (1), 65-75 
SAS (2003),PRINQUAL procedure, http://support.sas.com/documentation/cdl/en/statug/6 3347/HTML/default/viewer.htm\#prinqual_toc.htm

SAS (2003),PRINCOMP procedure, http://support.sas.com/documentation/cdl/en/statug/6 3347/HTML/default/viewer.htm\#princomp_toc.htm

Yang L., Hou L., Li H., Xu X., Liu J.(2015). Regression models for energy consumption prediction in airconditioned office building. J. Xi' an Univ. of Arch. \& Tech. (Natural Science Edition) 5, 707-711

Zhao H., Magoulès F.(2010), Feature Selection for Predicting Building Energy Consumption Based on Statistical Learning Method. Journal of Algorithms \& Computational Technology 1 (6), 59-78

Zhu M., Pan Y., Huang Z. (2018), A hybrid space filling design method of building performance database construction for office building energy prediction. Proceedings of ASim2018: IBPSA Asia Conference, Hong Kong, China, December 3-5

Zhao L., Liu J., Wang X. (2016), Hellinger distance based similarity analysis for categorical variables in mixture dataset. Computer Science 43(6), 280-282

Zhang X.(2016), PCA of mixed data - An example of statistical survey data in Gironde. [Master Thesis]. Jinan University

Zhou Z. (2016), Mechine Learning, Tsinghua University Press. Beijing (China). 